\title{
El control glucémico mejora la calidad de vida y la productividad de los pacientes con diabetes tipo 2.
}

Health Economic Benefits and Quality of Life During Improved Glycemic Control in Patients With Type 2 Diabetes Mellitus. A Randomized, Controlled, Double-Blind Trial. MA. Testa; DC Simonson. JAMA 1998; 280: 1490-96.

\section{Objetivo}

Evaluar si el mejor control glucémico en el corto plazo influye sobre la calidad de vida de pacientes con diabetes de tipo 2 y sobre diferentes parámetros económicos relacionados con su evolución.

\section{Diseño}

Ensayo clínico, randomizado a doble ciego comparando los efectos del buen control metabólico entre pacientes diabéticos tratados con placebo versus otros "tratados activamente" (glipizida de liberación controlada).

\section{Lugar}

2 centros de los Estados Unidos.

\section{Pacientes}

Se incluyeron 569 pacientes con Diabetes tipo 2, de ambos sexos, tratados mínimamente durante 3 meses con dieta o dieta y sulfonilureas. Se excluyeron pacientes embarazadas, con peso $<80 \% 0>160 \%$ del ideal, o insulinizados por más de 1 semana en los últimos 3 meses, con cetoacidosis en el año previo, o con historia de abuso de medicamentos o drogas.

\section{Intervención}

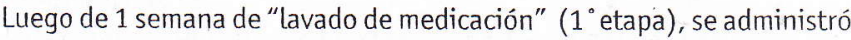
placebo a todos (simple ciego) durante 3 semanas ( $2^{\circ}$ etapa), randomizándolos luego (doble ciego) en 2 grupos: a) los que serían tratados con dieta (y placebo) y b) los que recibirían dieta y glipizida de liberación controlada (dosis inicial de $5 \mathrm{mg}$ /día que sería incrementada a 10 y 15 $\mathrm{mg} /$ día si las glucemias matinales fueran $>115 \mathrm{mg} / \mathrm{dl}$ ) manteniéndose este período 3 semanas ( $3^{\circ}$ etapa). La $4^{\circ}$ etapa (de estabilidad terapéutica) se mantuvo durante 8 semanas.

\section{Medición de resultados principales}

Se evaluaron al inicio y durante el seguimiento: a) El grado de control metabólico mediante la $\mathrm{HbA1c}$, glucemia de ayunas, insulinemia basal, péptido ( basal, lípidos, microalbuminuria; b) Cuestionarios de calidad de vida validados que incluyeron los siguientes aspectos: calidad de vida global, percepción de salud general, salud mental y emocional, función cognitiva autorreportada, y las molestias debido a los síntomas; c) La in- fluencia del tratamiento sobre los costos relacionados a la salud y de las ganancias perdidas. Se evaluaron días no trabajados por motivos de salud, días con actividad restringida y utilización del sistema de salud.

\section{Resultados principales}

Luego de 12 semanas se observó un descenso significativo de las $\mathrm{HbA} 1 \mathrm{c}$ en el grupo glipizida en relación al grupo placebo $(7.5 \%+/-0.1 \%$ vs $9.3 \%+/-0.1 \%$ y y también de las glucemias matinales $(126+/-2 \mathrm{mg} / \mathrm{dL}$ vs $168+/-4 \mathrm{mg} / \mathrm{dL})(\mathrm{P}<0.001$ para ambas comparaciones $)$. Esto resultó en un mejor control glucémico global significativo para el grupo glipizida. Este grupo también mejoró significativamente síntomas relacionados con hiperglucemia: poliuria $(P=0.048)$, visión borrosa $(P=0.049)$, síntomas de hiperglucemia $(p=0.05)$, y funciones cognitivas $(P=0.007)$. Las escalas de calidad de vida demostraron cambios más favorables para el grupo en tratamiento con glipizida $(P=0.008)$. Los beneficios más significativos se observaron en la percepción de síntomas, percepción de la salud, habilidades cognitivas, y calidad de vida global (oscilaron en +0.24 y +0.59 desvíos estándares). Los pacientes con glipizida disminuyeron significativamente los días de enfermedad ( $\mathrm{P}=0.02)$, días con actividad restringida $(\mathrm{P}<.001)$, y los días con limitación de actividades $(\mathrm{p}<.006)$. También mantuvieron más el empleo que los del grupo placebo ( $97 \%$ vs. $85 \%$, p 0.001). Se observó una reducción en el uso de servicios médicos $(\mathrm{p}=0.01)$, y menores tasas de internación. El análisis económico también favoreció al grupo glipizida en relación al placebo: menores pérdidas mensuales por ausentismo, U\$24 vs U\$115/ pac $(\mathrm{P}<0.001)$, menores costos por internación, $U \$ 1539$ vs $\$ 1843$ por cada 1000 personas-días $(\mathrm{P}=0.05)$, menor costo por menos días de actividad restringida, $U \$ 2660$ vs $U \$ 4275$ por cada 1000 personas-días; $(P=0.01)$ y menos gastos del sistema médico (reducción de U\$ 11 por paciente y por mes).

\section{Conclusiones}

El mejor control glucémico en pacientes con Diabetes Mellitus tipo 2 se asocia, en este estudio con seguimiento a corto plazo, con una significativa mejoría en los índices de calidad de vida y resulta en un beneficio sobre los costos relacionados con la salud de estos pacientes y con su capacidad productiva.

Fuente de financiamiento: Agency for Health Care Policy and Research, American Diabetes Association, EE.UU. Laboratorio Pfizer.

\section{COMENTARIO}

Si bien existen trabajos que demuestran la mejoría en la calidad de vida y la disminución y mejor aprovechamiento de los costos directos e indirectos relacionados con el control metabólico de la Diabetes tipo 2, la mayoría de ellos consideró períodos prologados de tiempo. Los resultados en lapsos cortos son aún controvertidos. Sin embargo este trabajo, que se realizó durante un protocolo farmacológico, tiene un diseño muy atractivo ya que consideró la evolución de diferentes parámetros de la vida cotidiana (subjetivos) y datos objetivos relacionados con los costos de esta enfermedad. La tendencia actual en la investigación clínica es darle importancia no sólo a los resultados 'duros' y objetivos (como las complicaciones vasculares o la glucemia), sino también a resultados relacionados con la vida cotidiana y percepciones subjetivas de los propios pacientes (como percepción de la salud, o molestias por síntomas). Queda demostrado que un buen control glucémico se acompaña de una mejoría en la calidad de vida (mejoría en los síntomas relacionados con la hiperglucemia, menor pérdida en la capacidad laboral y productiva) y también de un menor uso de los recursos del sistema médico. Debemos agregar además que si esta situación se mantiene en el largo plazo también se reducirá la prevalencia y evolución de algunas complicaciones tardías, como demostraron los estudios $\mathrm{DCCT}^{1}$ en diabetes tipo 1 y $U_{K P D S}^{2}$ en diabetes tipo 2. Al analizar los costos hay que considerar que parte de ellos (ahorrados) deberán ser utilizados para lograr normoglucemia (medicación, tirillas y educación diabetológica). Surge de este trabajo que el buen control metabólico, al beneficiar subjetiva y objetivamente al paciente con diabetes en el corto plazo, sería un factor muy importante para motivar a éste en sostener su tratamiento durante períodos más prolongados de tiempo, hecho necesario si se quiere influir sobre la aparición y evolución de las complicaciones diabéticas crónicas, sobre todo microangiopáticas.

\section{Dr. León E. Litwak}

Servicio de Endocrinología, Metabolismo y Medicina Nuclear. Hospital Italiano de Buenos Aires.

\section{Referencias}

1. The DCCT Research Group. The effect of intensive tratment of Diabetes on the development anc progression of long term complications in insulin dependent diabetes mellitus. 